\title{
NOTE
}

\section{Equivalent Responses to Lexical and Nonlexical Visual Stimuli in Occipital Cortex: A Functional Magnetic Resonance Imaging Study}

\author{
Peter Indefrey,*,1 Andreas Kleinschmidt, † Klaus-Dietmar Merboldt,† Gunnar Krüger, † Colin Brown,* \\ Peter Hagoort,* and J Ens Frahm $\dagger$
}

*Max-Planck-Institut für Psycholinguistik, PB 310, 6500 AH Nijmegen, The Netherlands; and †Biomedizinische NMR Forschungs GmbH am Max-Planck-Institut für BiophysikalischeChemie, PB 2841, 37018 Göttingen, Germany

Received J une 3, 1996

Stimulus-related changes in cerebral blood oxygenation were measured using high-resolution functional magnetic resonance imaging sequentially covering visual occipital areas in contiguous sections. During dynamic imaging, healthy subjects silently viewed pseudowords, single false fonts, or length-matched strings of the same false fonts. The paradigm consisted of a sixfold alternation of an activation and a control task. With pseudowords as activation vs single false fonts as control, responses were seen mainly in medial occipital cortex. These responses disappeared when pseudowords were alternated with false font strings as the control and reappeared when false font strings instead of pseudowords served as activation and were alternated with single false fonts. The string-length contrast alone, therefore, is sufficient to account for the activation pattern observed in medial visual cortex when word-like stimuli are contrasted with single characters. $\odot$ 1997 Academic Press

\section{INTRODUCTION}

Areas of the visual cortex have been reported to be specifically activated by word-like stimuli (Petersen et al., 1990). The data leading to this conclusion were obtained in a PET experiment in which words, wordlike stimuli, i.e., pseudowords, and nonlexical stimuli, i.e., consonant strings and false font strings, were visually presented with a frequency of $1 / \mathrm{s}$ and a duration of $150 \mathrm{~ms}$. Only the lexical stimuli evoked significant activation in the left medial extrastriate cortex when contrasted with a fixation cross-stimulus serving as the baseline condition for all stimuli. These findings,

\footnotetext{
${ }^{1}$ To whom correspondence should be addressed. Fax: +31-243521213; E-mail: indefrey@mpi.nl.
}

however, could not be reproduced in a subsequent PET study with longer (1000 ms) stimulus duration and length-matched strings of false fonts as the baseline condition (Howard et al., 1992). Stimulus duration was ruled out as a variable accounting for these differences by comparing short and long stimulus durations (Price et al., 1994). With false font strings as the baseline condition no additional activation for words was found in the critical area for any of the two durations. Conversely, with fixation cross-control both words and pseudowords induced bilateral medial occipital activation in a PET study using long (758 ms) stimulus durations (Indefrey et al., 1995). This pattern of results suggests that it was not the lexical status of word-like stimuli, but rather their length or visual complexity that was associated with medial extrastriate activation. The aim of the present functional magnetic resonance imaging study was to explicitly test this hypothesis with respect to the string-length contrast.

\section{MATERIALS AND METHODS}

Eight heal thy malesubjects participated in theexperiment. They were all right-handed, as assessed by an adapted German version of the Edinburgh inventory, and native speakers of German. All subjects underwent functional mapping at $2 \mathrm{~T}$ (Siemens Magnetom) using the standard imaging headcoil and rf-spoiled FLASH $\mathrm{MRI}\left(\mathrm{TR} / \mathrm{TE}=62.5 / 30 \mathrm{~ms}\right.$, RF flip angle $10^{\circ}$, voxel size $0.8 \times 1.6 \times 4.0 \mathrm{~mm}$; see Frahm et al., 1994). Recordings were performed in four contiguous 4-mm sections parallel to the bicommissural plane (eight sections for three of the subjects). I n six of eight subjects the ventral occipital cortex was entirely covered. In five of eight subjects the occipital cortex at and above AC-PC level was included. The order of serial volume coverage by single sections as well as presentation of the stimuli were counterbalanced across subjects. Each dynamic 
series comprised six cycles of activation (18 s) and control (36 s) with continuous stimulus projection at a fixed rate (1/1.5 s, stimulus duration $750 \mathrm{~ms})$, and subjects were instructed to silently regard. Responses were determined by pixel-based temporal correlation with a reference boxcar waveform shifted by one data point relative to the protocol. Activation sites in these correlation maps were identified by analyzing the distribution of correlation coefficients in tissue pixels. While in the absence of stimulation this distribution is symmetrical and approximately Gaussian, correlated activation introduces a deviation from symmetry at high positive correlation coefficient values. By assuming a noise distribution and computing its integral, each correlation coefficient value was transformed into an error probability for the hypothesis that a pixel achieving this value in that particular experiment was activated. To account for the nonspecific effect of intertrial variability in distribution broadness, the noise distribution was obtained from a Gaussian fit to the negative and central part of the distribution in each individual experiment. Thresholding was performed at an error probability level of $0.05 \%$ corresponding to approximately 10 false positive pixels maximally admitted per map. Delineation of response areas was improved by iteratively incorporating neighboring pixels into these foci if their correlation coefficients were high enough to contribute to the deviation of the actual distribution from the noise distribution. Performance of data analysis procedure has been described in detail elsewhere (Kleinschmidt et al., 1995). The resulting map of correlation coefficients was color-coded and overlaid on a corresponding flow-sensitized anatomic image (TR/TE $=70.3 / 7.5, \mathrm{RF}$ flip angle $60^{\circ}$ ) of identical spatial resolution.

The stimuli used were pseudowords, single false fonts, and strings of the same fal se fonts matched to the pseudowords for length and length variation. The pseudowords were created from real words by replacing at least two letters. The false fonts were created by recombination of character elements (ascending, descending, horizontal, and curved lines) of the font type, Arial, which was also used for the presentation of the pseudowords. Table 1 shows the design of the experimental sessions. In the maximal contrast condition, pseudowords as activation were alternated with single false fonts as control. In the dimination condition, false font strings, instead, served as control stimuli. Since they were strings of the same length as the pseudowords, this condition was intended to eliminate that
TABLE 1

Experimental Design

\begin{tabular}{|c|c|c|c|}
\hline \multirow[b]{2}{*}{ Condition } & \multicolumn{3}{|c|}{ Category, example } \\
\hline & Activation & & Control \\
\hline Maximal contrast & $\begin{array}{l}\text { Pseudowords } \\
\text { DE B A M }\end{array}$ & vs & $\begin{array}{c}\text { Single false font } \\
\mathbb{P}\end{array}$ \\
\hline Elimination & $\begin{array}{l}\text { Pseudowords } \\
\text { DE B A M }\end{array}$ & vs & $\begin{array}{c}\text { False font strings } \\
\text { PPPP/P }\end{array}$ \\
\hline I solation & $\begin{array}{c}\text { False font strings } \\
\text { P/P/P/P }\end{array}$ & vs & $\begin{array}{c}\text { Single false font } \\
\mathbb{P}\end{array}$ \\
\hline
\end{tabular}

part of the maximal signal that was due to the stringlength contrast. By "string length" wemean the conjunction of three properties of strings: to be horizontally extended, to vary in horizontal extension, and to be composed of subunits. In the isolation condition, false font strings served as activation and were alternated with single false fonts.

\section{RESULTS}

The strongest responses to the maximal contrast condition were observed in medial occipital cortex (Fig. 1, top row). Depending on the individual anatomy, activations were located ventrally in the lingual, posterior fusiform, and parahippocampal gyri. Dorsally, they were located in the cuneus and the cal carine cortex. All these occipital responses to pseudowords were cancel ed by alternating their presentation with false font strings as control (Fig. 1, middle row). An activation pattern identical to that seen with pseudowords as activation reappeared when false font strings as activation were alternated with single fal se fonts during control (F ig. 1, bottom row). This sequence of occipital signal extinction and reappearance was seen in all subjects. Additional responses were observed in some subjects at the occipitotemporal junction and along the posterior superior temporal sulcus. These were lateralized to the left and behaved differently, in that they were not canceled by false font strings and did not appear in the complementary condition with false font strings as activation.

\section{DISCUSSION}

This study demonstrates that string-length differences account for activations in visual occipital areas

FIG. 1. Data from two subjects (left and right columns) in the section $4 \mathrm{~mm}$ above the individually determined bicommissural plane. Rows correspond to conditions listed in Table 1, i.e., maximal contrast (top row), elimination (middle row), and isolation (bottom row), and are indicated by insets of stimulus examples. Color coding of activation maps ranges from yellow (highest correlation coefficient observed) to red (lowest correlation coefficient admitted by the analysis procedure). $\mathrm{R}$, right side. 

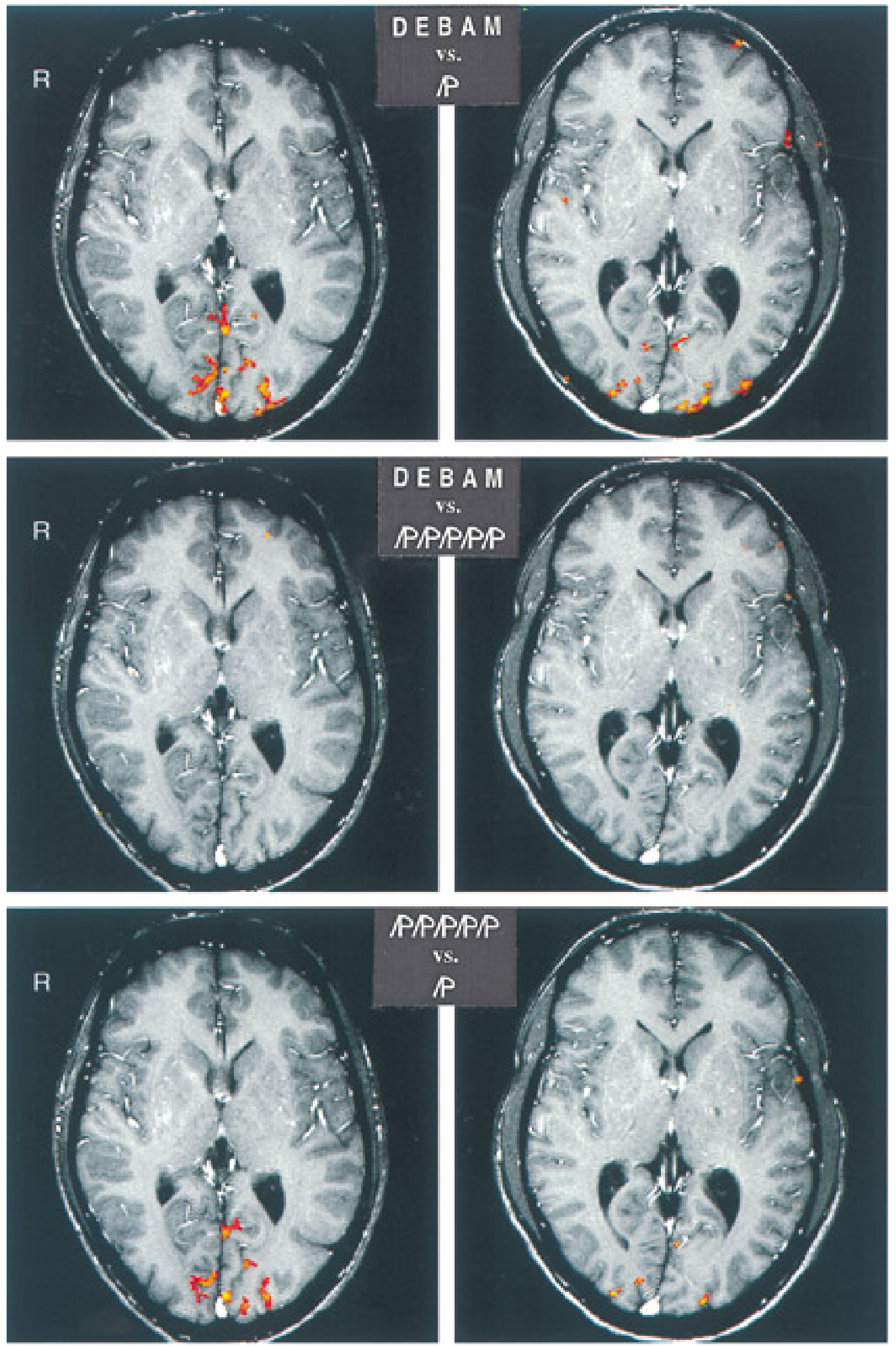
reported previously to be induced by lexical visual stimuli when contrasted with fixation cross-presentation (Petersen et al., 1988, 1990; I ndefrey et al., 1995). In the maximal contrast condition, comparing the silent viewing of pseudowords vs single false fonts, we replicated the "medial extrastriate activation" first reported by Petersen et al. (1990). Our measurement volume covered the activated area shown in their Fig. 1C, and included the level of the activation focus reported for pseudowords $(z=+4)$ for five of eight subjects. Our finding that this activation disappears with false font strings as control is in line with previous PET studies (Howard et al., 1992; Price et al., 1994, 1996). Price et al. (1996), however, do report more posterior medial "cuneus (extrastriate cortex)" activation in a feature detection task contrasting lexical stimuli with length-matched false font strings as controls. This activation was located at a considerable distance from the region reported by Petersen et al. (1990) and in the immediate vicinity of a striate cortex activation found in Price et al. (1994) for the same control task. The absence of this activation in the silent viewing experiment of Price et al. (1994) and in our data, which were also obtained for silent viewing, suggests that it is not due to a lexicality effect but rather to feature detection as an additional top-down task component that may differentially affect the perception of false fonts and word-like stimuli. Our study differs from the previous studies in one important respect: the false font strings we used were matched only in length and length variation, and had no variability in their characters. Not just the potential lexicality of pseudowords but also their greater visual complexity due to different letter shapes can therefore be ruled out as being correlated with the medial occipital signal.

There remains al ways a possibility that methodological shortcomings, such as insufficient sensitivity or an increase in noise level, might be responsible for negative results. We tested this by isolating the string-length contrast. To the extent that the absence of a signal in the elimination condition was really due to the shared stringlength features of activation and control, the signal could be predicted to reappear in this condition. Reappearance of the complete signal confirmed that it was indeed the string-length contrast that was reflected in the occipital response to the maximal contrast condition.

It remains to be explained why Petersen et al. (1990) obtained negative results for their nonlexical string vs fixation cross-conditions, and found left-lateralized activations in the lexical conditions instead of the bilateral activations we found in the present study and in previously reported PET data (Indefrey et al., 1995). The finding of left-lateralized activations for words provides an indication that fluctuations in noise level might have played a role. In an earlier PET study, Petersen et al. (1988) obtained bilateral responses for the word vs fixation cross-comparison, with right-sided responses being weaker. The failure of such responses to pass the statistical threshold in the later study indicates that also medial extrastriate responses in the nonlexical string conditions might have just failed to become significant. I mages $1 \mathrm{~A}$ and $1 \mathrm{~B}$ of Petersen et al. (1990) suggest at least some activation in this area.

In our study, the measurement volume was intended to cover occipital rather than temporal cortex. This may in part explain why left posterior temporal activations were only inconsistently found. These activations may correspond to the "lateral extrastriate" activations reported without giving the exact location by Petersen et al. (1990) not only for word-like stimuli but also for consonant and (heterogeneous) false font strings when contrasted with fixation cross-control. Similar activations have been reported by Howard et al. (1992) and Price et al. (1994, 1996) for words and pseudowords with length-matched control stimuli. Since these activations did not disappear when control stimuli were matched for length, they cannot be related to the string length of visual stimuli, but may rather beassociated with some higher order aspect of visual stimulus complexity.

\section{CONCLUSIONS}

Our data show that medial occipital responses to visual lexical stimuli areassociated with the processing of their string length. At the same time we failed to find specifically lexical activation or activation related to character variability in this region. The present data suggest, ther efore, that the medial occipital cortex does not specifically subserve visual lexical processing.

\section{REFERENCES}

Frahm, J ., Merboldt, K.-D., Hänicke, W., Kleinschmidt, A., and Boecker, H. 1994. Brain or vein-Oxygenation or flow? On signal physiology in functional MRI of human brain activation. NMR Biomed. 7:45-53.

Howard, D., Patterson, K., Wise, R., Brown, W. D., Friston, K., Weiller, C., and Frackowiak, R. 1992. The cortical localization of the lexicons. Brain 115:1769-1782.

Indefrey, P., Hagoort, P., Brown, C., Law, I., Herzog, H., Steinmetz, H., and Seitz, R. J . 1995. The reading of words and legal nonwords: A [ $\left.{ }^{15} \mathrm{O}\right]$-butanol PET study. Hum. Brain Map. Suppl. 1:220.

Kleinschmidt, A., Requardt, M., Merboldt, K.-D., and Frahm, J . 1995. On the use of temporal correlation coefficients for magnetic resonance mapping of functional brain activation: Individualized thresholds and spatial response delineation. Int. J . I mag. Syst. Tech. 6:238-244.

Petersen, S. E., Fox, P. T., Posner, M. I., Minton, M., and Raichle, M. E. 1988. Positron emission tomographic studies of the cortical anatomy of single-word processing. Nature 331:585-589.

Petersen, S. E., Fox, P. T., Snyder, A. Z., and Raichle, M. E. 1990. Activation of extrastriate and frontal cortical areas by visual words and word-like stimuli. Science 249:1041-1044.

Price, C. J ., Wise, R. J . S., Watson, J . D. G., Patterson, K., Howard, D., and Frackowiak, R. S. J . 1994. Brain activity during reading: The effects of exposure duration and task. Brain 117:1255-1269.

Price, C. J ., Wise, R. J . S., and Frackowiak, R. S. J . 1996. Demonstrating the implicit processing of visually presented words and pseudowords. Cereb. Cortex 6:62-70. 